\title{
Linear estimation discriminates midline sources and a motor cortex contribution to the readiness potential
}

\author{
Thomas Knöschea,*, Peter Praamstra ${ }^{b}$, Dick Stegeman ${ }^{b}$, Maria Peters $^{a}$ \\ ${ }^{a}$ Faculty of Applied Physics, Low Temperature Division, Biomagnetic Centre, University of Twente, P.O. Box 217, 7500 AE Enschede, The Netherlands \\ bInstituie of Neurology, University of Nijmegen, P.O. Box 9101, 9500 HB Nijmegen, The Netherlands
}

Accepted for publication: 3 April 1996

\begin{abstract}
Spatiotemporal dipole modelling of the generators of the readiness potential (RP) prior to voluntary movements has yielded diverging results concerning the contributions of supplementary motor area (SMA) and primary motor cortex. We applied an alternative approach (i.e. linear estimation theory) to measurements of the RP preceding fixed and freely selected finger movements, measured at 28 electrodes of the extended 10-20 system. The volume conductor properties of the head were modelled by 3 concentric spheres. Current densities were reconstructed on a spherical surface, placed at a depth of $5 \mathrm{~mm}$ from the inside of the skull. Lead field normalization was applied. The analysis shows activity on the midline as well as near the primary motor area. Although some features of the reconstructions are not readily interpretable, separate contributions of midline sources (including presumably SMA) and motor cortex to the RP are clearly distinguished.
\end{abstract}

Keywords: Readiness potential; Linear estimation; Supplementary motor area

\section{Introduction}

It is widely agreed that the application of dipole source analysis to brain potentials recorded from the scalp requires a considerable amount of prior knowledge regarding the brain structures that might be involved in their generation (e.g., Scherg and Berg, 1991; George et al., 1995).The readiness potential (RP) is probably among the potentials that best fulfil this precondition, as there is a large body of evidence on movement-related cerebral activity, obtained with a range of different techniques. Paradoxically, source analysis of the RP using a spatiotemporal dipole source analysis approach has recently suggested that one of the putative generators of the premovement negativity of the RP, the role of which seemed well established, i.e. the supplementary motor area (SMA) (for a review see Deecke, 1987), does not contribute to the RP (Bötzel et al., 1993; Toro et al., 1993; Böcker et al., 1994). However, using the same spatiotemporal dipole source approach, Praamstra et al. (1996) have presented an alternative model that does include an

* Corresponding author. Tel.: +31 53 4892938; fax: +31 534891099 .
SMA contribution to the premovement negativity of the RP. The goal of the present study is to evaluate the diverging results by applying a linear estimation approach to the same data as analyzed by Praamstra et al. Linear estimation is a method to reconstruct brain activity from electroencephalographic (EEG) and/or magnetoencephalographic (MEG) measurements. It produces tomography-like pictures of the current density distribution within the brain and is capable of reconstructing distributed sources. One important advantage is that the solution does not require a non-linear optimization process and therefore cannot be trapped in local minima of the goal function. In the present paper linear estimation was used to reconstruct the sources of the RP on a spherical surface underneath the skull.

\section{Methods}

\subsection{Experimental design and data acquisition}

Methods other than source reconstruction are described briefly. For a more comprehensive description, we refer to Praamstra et al. (1995). 


\subsubsection{Tasks}

Movement-related potentials were recorded preceding finger movements. Subjects made flexion movements of the index or the middle finger of the left or the right hand by pressing one of 4 response buttons on which the fingers were resting. The experiment consisted of 8 experimental blocks of $6 \mathrm{~min}$ duration each. There were two types of blocks. In the 4 'fixed' blocks subjects pressed a designated button at a self-paced rate of once every 5$10 \mathrm{~s}$. In the 4 'free' blocks subjects could on each trial freely select one of the 4 movement alternatives. Fixed and free blocks alternated. Half the subjects began with a fixed and half with a free block.

\subsubsection{Subjects}

Sixteen right-handed subjects ( 7 men and 4 women) participated in the experiment. The subjects' mean age was $27 \pm 5$ years (range $21-37$ years).

\subsubsection{Electrophysiological recording and data processing}

The EEG was recorded with $\mathrm{Ag} / \mathrm{AgCl}$ electrodes placed at the midline sites $\mathrm{Fz}, \mathrm{Cz}$, and at 26 lateral sites, F3 and F4, F1 and F2, FC5 and FC6, FC3 and FC4, FC1 and $\mathrm{FC} 2, \mathrm{C} 5$ and $\mathrm{C} 6, \mathrm{C} 3$ and $\mathrm{C} 4, \mathrm{C1}$ and $\mathrm{C} 2, \mathrm{CP} 5$ and CP6, CP3 and CP4, CP1 and CP2, P3 and P4, P1 and P2. All electrodes were referenced to linked mastoids. Vertical and horizontal EOG were recorded bipolarly from above/below the right eye and from the outer canthi of each eye. Electrode impedance was kept below $5 \mathrm{k} \Omega$. EMG activity was recorded bipolarly with electrodes attached $8 \mathrm{~cm}$ apart to the flexor side of each forearm. EEG activity was amplified using a bandpass of $0.016-35 \mathrm{~Hz}$ (EMG $10-70 \mathrm{~Hz}$ ) and digitized at a rate of 200 samples/ s. Movement of each of the 4 buttons caused the closure of a switch, which delivered a trigger pulse used for averaging. Individual trials were checked visually prior to averaging and trials contaminated by artifacts (e.g. due to excessive eye movements or amplifier saturation) were rejected. Electrical activity was averaged for an analysis period of $2750 \mathrm{~ms}$ starting $2250 \mathrm{~ms}$ prior to movement onset. The baseline was calculated from the first $250 \mathrm{~ms}$.

Basic data processing yielded 4 sets of waveforms for each subject, namely, RPs preceding fixed and freely selected movements with the left and the right hand. Fig. 1 shows the superimposed individual data of the 16 subjects for right hand movements at selected scalp locations. Two additional sets of data were created by averaging RPs for left and right hand movements. This was done after interchanging the left and right hemisphere potentials for the RPs preceding left hand movements, in order to preserve the relation between scalp topography and side of movement. These two additional data sets (for fixed and freely selected movements, respectively) will be further referred to as left/right averages. Finally, another two sets of data were created by averaging the RPs for fixed and freely selected movements. These data will be referred to as
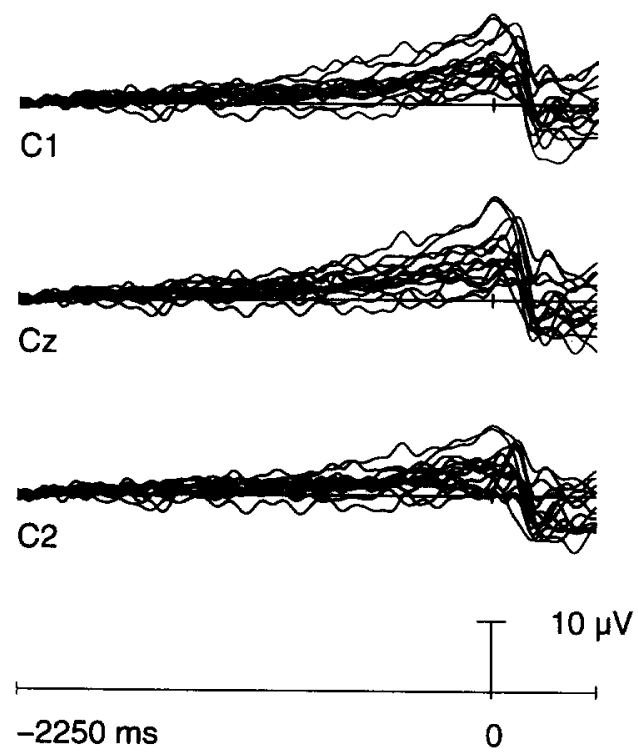

Fig. 1. RPs recorded at central electrode sites $\mathrm{C1}, \mathrm{Cz}$, and $\mathrm{C2}$, superimposed for all subjects. The data represent activity preceding freely selected movements of the right hand.

fixed/free averages (one set for right hand movements and one set for left hand movements). For each of the 8 data sets grand averages were created across subjects. For all scalp locations the waveforms of the left/ right averages are depicted in Fig. 2. Part of the data are represented as potential maps in Fig. 3.

\subsection{The linear estimation method}

Linear estimation is a technique to reconstruct distributed or possibly distributed sources of EEG or MEG, where the solution is geometrically confined to a certain region (Dale and Sereno, 1993; Fuchs et al., 1993; Wang, 1994). A reconstruction region that can be a volume or a surface is chosen and discretized into current elements. These current elements are similar to the current dipoles used in traditional dipole fit analysis. However, their positions are fixed, and only their strengths are fitted to the data. Since the strength parameters have only linear influence on the EEG, the vector of dipole magnitudes $\boldsymbol{q}$ is mapped to the vector of (simulated) potentials $\varphi$ by means of the lead field matrix $L$ :

$$
\varphi=\mathbf{L} \cdot \mathbf{q}
$$

Since there are many more dipole strength parameters than there are measurement values (the lead field matrix is not square), an infinite number of (similar) solutions for $\varphi$ is possible. To pick from this manifold one representative, we use the so-called minimum norm (MN) condition, i.e. the one solution with the minimum sum of square dipole magnitudes is taken. Since this, however, would favour such dipoles that cause a relatively large 
$\overline{\text { Heog }} \widetilde{\text { veog }}$
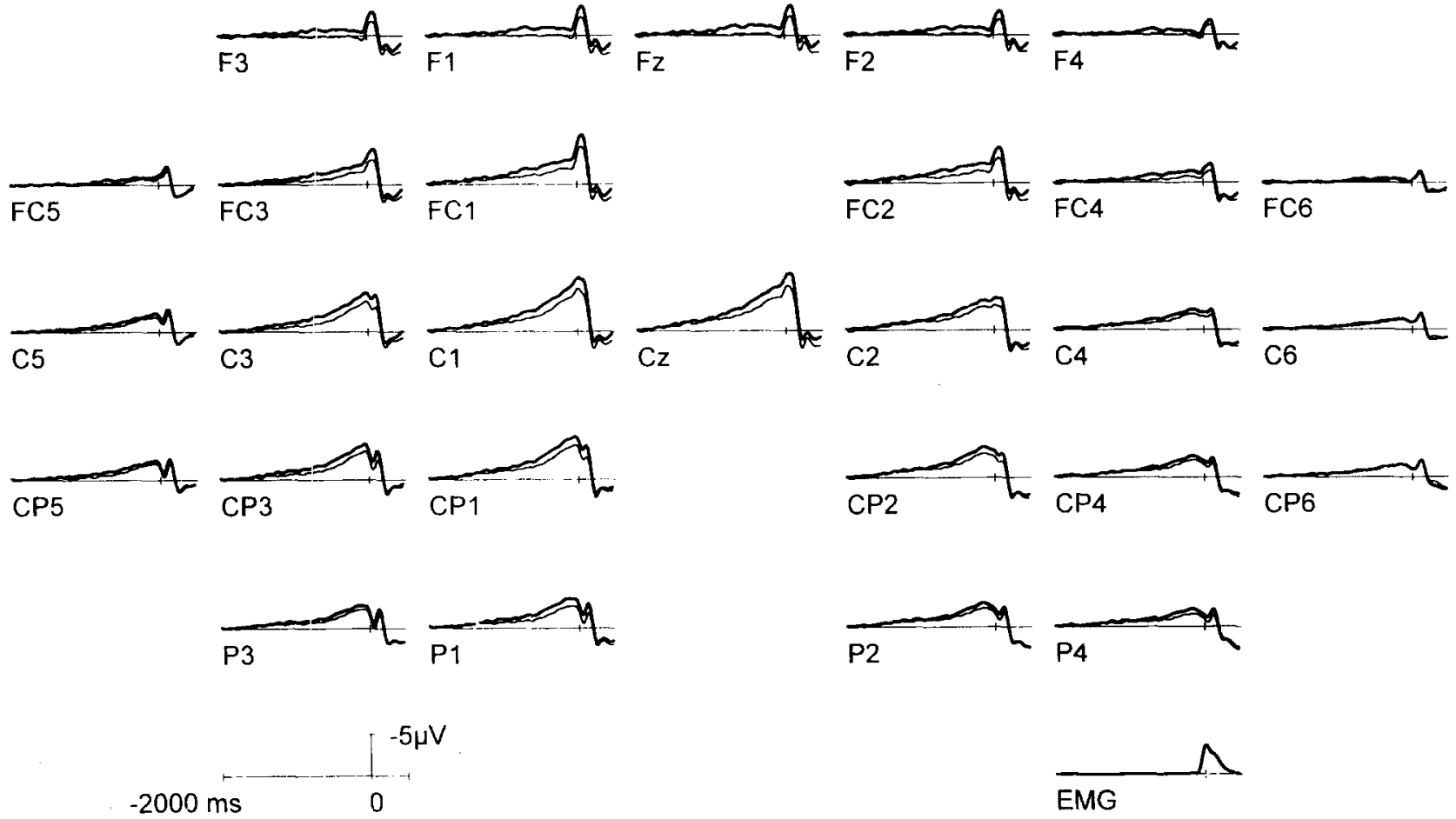

Fig. 2. Superposition of grand average RPs preceding fixed (thin line) and freely selected movements (thick line). The lay-out of the traces reflects the arrangement of electrodes on the subjects' heads. EMG is displayed in the lower right corner. The data represent RPs for left and right hand movements, which were averaged after transferring the left and right hemisphere potentials to the opposite hemisphere for the RPs preceding left hand movements. This preserved the scalp topography with higher amplitudes over the hemisphere contralateral to the hand that moves.

EEG with comparatively little strength (e.g. those directly underneath the electrodes), a compensation, called lead field normalization (LFN), is applied.

The MN condition picks the (in some sense) smoothest of all possible solutions. The reason for this is that, if the activity of one dipole is distributed evenly over two dipoles to cause the same EEG, the two dipoles would both need approximately half the strength of the original one, while their sum of squares becomes considerably smaller (if $a+b=c$ then $a^{2}+b^{2}<c^{2}$ ). To obtain a sharper solution, an iterative procedure can be utilized (see Gorodnitsky et al., 1992). The lead field matrix is weighted using the previous solution, favouring strong dipoles and suppressing weak ones. Applying this repeatedly, a concentration or sharpening effect can be achieved. This is not a better solution in terms of explaining the data, but it can make things more clear.

As source space, we use a hemispherical surface with equidistantly distributed dipole positions. At each position three orthogonal dipoles are placed. The hemisphere is placed at a certain depth underneath the innermost shell of the volume conductor model, representing the inside of the skull. One consequence of this source space is that all activity will be projected onto one surface. Thus, no depth information can be obtained.

\subsection{Data analysis}

The 8 grand average data sets including left/right averages for fixed and freely selected movements as well as the fixed/free averages for left and right hand action were analyzed within an interval from $250 \mathrm{~ms}$ before to $100 \mathrm{~ms}$ after movement onset. The head was modelled using 3 concentric spheres accounting for the inside of the skull (radius $88 \mathrm{~mm}$ ), outside of the skull (radius $93 \mathrm{~mm}$ ), and scalp surface (radius $100 \mathrm{~mm}$ ). The conductivities were assumed to be isotropic and were set to $0.33 \mathrm{~S} / \mathrm{m}$ for the brain, $0.0042 \mathrm{~S} / \mathrm{m}$ for the skull, and $0.33 \mathrm{~S} / \mathrm{m}$ for the scalp.

The source space formed a hemispherical surface with a radius of $83 \mathrm{~mm}$. Dipoles were placed on this surface at points with a mutual distance of approximately $1 \mathrm{~cm}$. At each position one was oriented into $\theta$, one into $\alpha$, and one into $r$ direction. The 1377 strength parameters were fitted separately for each time sample using LFN. Alternatively, 5 iteration steps where used on top of LFN in order to 
sharpen the solution. After fitting, the dipoles belonging to one position were combined to one. For visualization of the dipole strengths, we interpolated the magnitudes of the resulting dipoles between the positions and plotted them in a combined contour-colour plot on the source space. Parallel projection was employed to map the curved source space onto the screen.

\section{Results}

The separate analyses of the grand averaged RPs for fixed and freely selected movements of the left and the right hand yielded consistent results. The main findings will be discussed on the basis of the results for the composite left/right averages and the fixed/free averages, as these data sets had the best signal-to-noise ratio.

\subsection{Left/right averages}

The results for the left/right averages are presented in Fig. 4a,b, showing the magnitude of the current density plotted for free and fixed movements, respectively. Fig. $4 \mathrm{c}, \mathrm{d}$ depicts the results achieved using 5 iteration steps. As the figure shows, there are 3 consistent foci of activity. One is located at the vertex, one at the frontocentral midline, and another at a lateral frontocentral location, approximately under electrodes C3 and FC3 contralateral to the side of movement. A small focus of activity at a parietal location is only present in the model for freely selected movements. It should be emphasized, however, that this focus seems not specific for the RP preceding freely selected movements, as it was present in the separately analyzed data for fixed as well as freely selected left hand movements, and was absent in the data for freely selected and fixed right hand movements. Differences between the models for the RP preceding fixed and freely selected movements are limited to more pronounced activity of the vertex focus with freely selected movements, most notably at times -50 and $0 \mathrm{~ms}$. Iterations of the fitting step reduces the number of active generators, represented as more localized foci in Fig. 4c,d. Iteration also caused the vertex focus and the lateral focus of activity to move towards each other.

\subsection{Fixed/free averages}

Analysis of the composite averages for left and for right hand movements showed the same configuration of active sources as described above. There was a major difference, however, between the source models for RPs preceding left and preceding right hand movements. Whereas for right hand movements only the contralateral MI was active (as was the case in the left/right averages shown in Fig. 4), with left hand movements the ipsilateral hemisphere also showed an active source (Fig. 5). Although this ipsilateral activity tends to be located more anteriorly than the contralateral activity, it presumably represents $\mathrm{MI}$.

\subsection{Midline activity: spurious or real?}

In order to rule out the possibility that the midline focus of current density is due to summation of bilateral motor cortex activity, we designed a simulation experiment. Using the same volume conductor model and electrode configuration as employed for the analysis of the measurements, we simulated EEG data from different pairs of dipoles. The two dipoles' positions and orientations were symmetrical with respect to the midline. We tested 3 different pairs of positions: between the electrodes $\mathrm{C5}$ and $\mathrm{C} 3$ (and $\mathrm{C} 6$ and $\mathrm{C} 4$ ), underneath $\mathrm{C} 3$ and $\mathrm{C} 4$, and between $\mathrm{C} 3$ and $\mathrm{C} 1$ (and $\mathrm{C} 4$ and $\mathrm{C} 2$ ), all at a depth of $5 \mathrm{~mm}$ beneath the innermost shell of the volume conductor model. The orientation we varied in steps of $45^{\circ}$ around all axes, resulting in 13 different configurations. The resulting EEGs were contaminated with white Gaussian noise of a level similar to that of the measurement data as estimated from the baseline interval (the first $250 \mathrm{~ms}$ ). Then, we applied our reconstruction method to it. In all of 39 tested cases, the reconstruction consisted of two lateral foci only, showing no spurious activity on the midline. For four selected cases, the reconstruction results are depicted in Fig. 6.

\section{Discussion}

\subsection{Comparison with previous source models of the RP}

Given the divergence between different spatiotemporal dipole source models that have been proposed for the RP, it was the goal of the present study to apply another source characterization approach for comparison. Results were not expected to resolve the discrepancies in a definitive way. Comparison of the results from different analyses could, nevertheless, provide support for one or another alternative. No less important, it provides an opportunity to compare the merits of the different source analyses.

There is no doubt that the present results are difficult to reconcile with spatiotemporal source models of the RP that do not include generators located near the midline, or that limit the contribution of such a source to the interval after movement onset. Our results consistently show active generators at midline locations as well as activity at a lateral location representing the motor cortex. Given that the non-realistic spherical head model used in our analyses does not allow very accurate localization, the two midline foci cannot be directly assigned to anatomical structures. The SMA is the midline structure most often considered as a possible generator of the RP (Deecke, 1987). However, positron emission tomography (PET) studies have revealed other midline structures to be active 

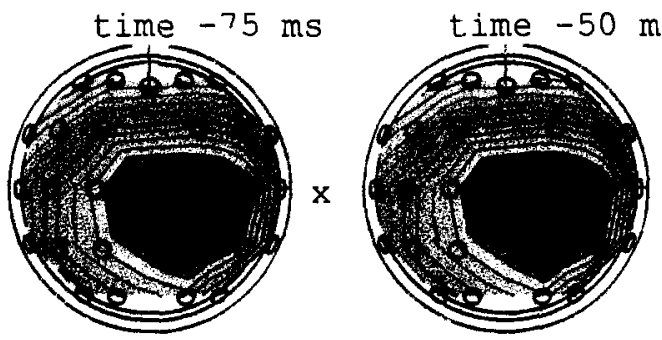

(a)
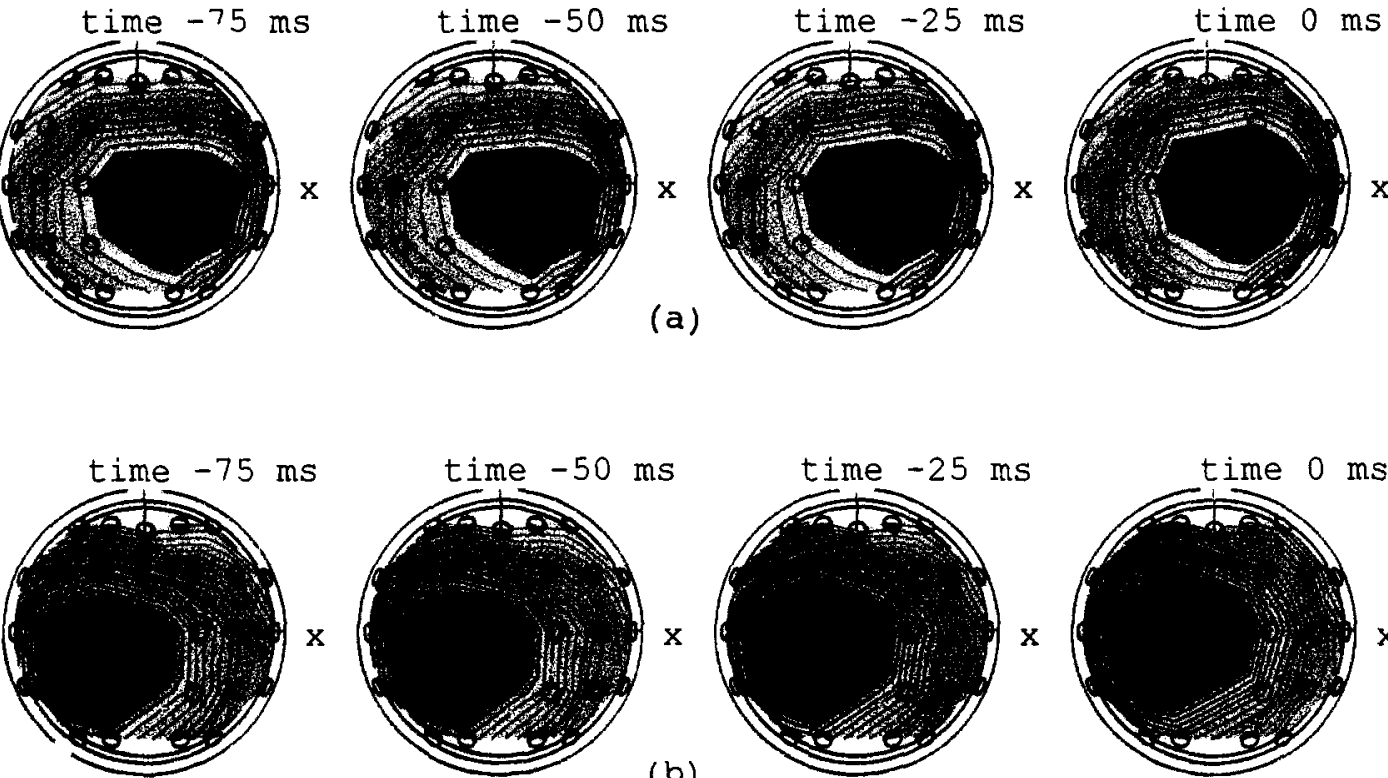

(b)

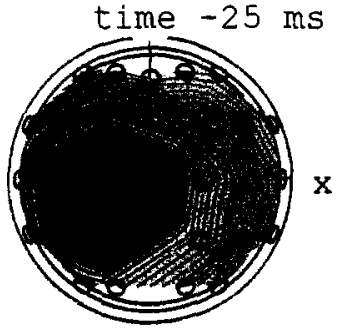

time $0 \mathrm{~ms}$
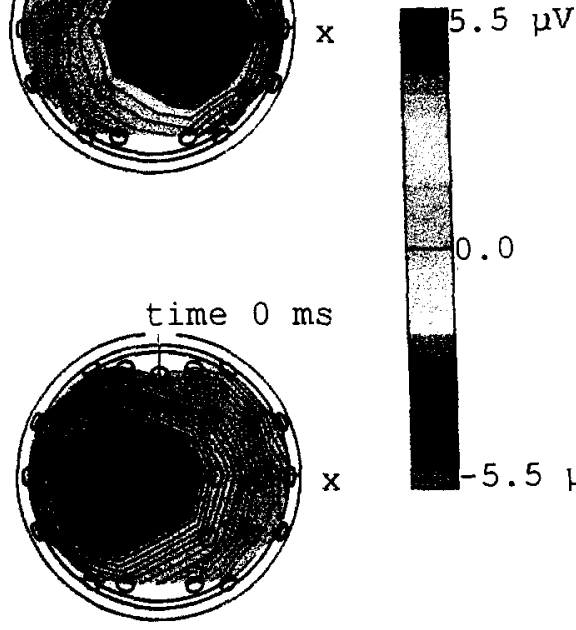

Fig. 3. Potential map of left hard (a) and right hand (b) freely selected movements before movement onset. Electrode positions are indicated by circlets.
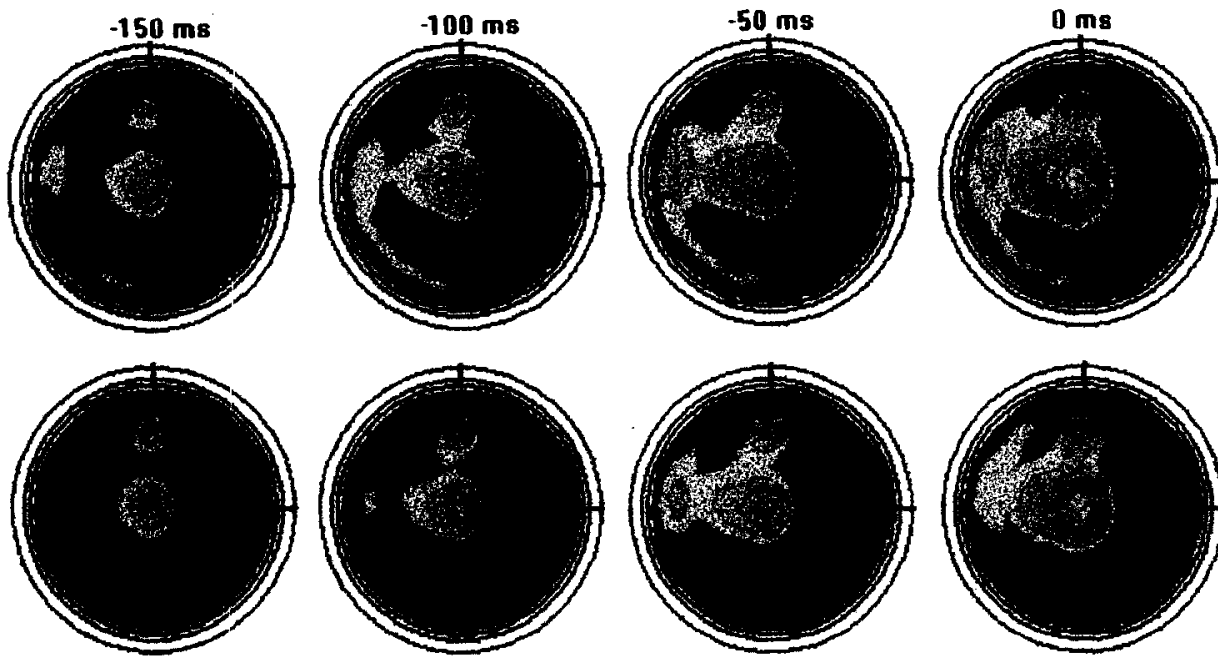

[a\}
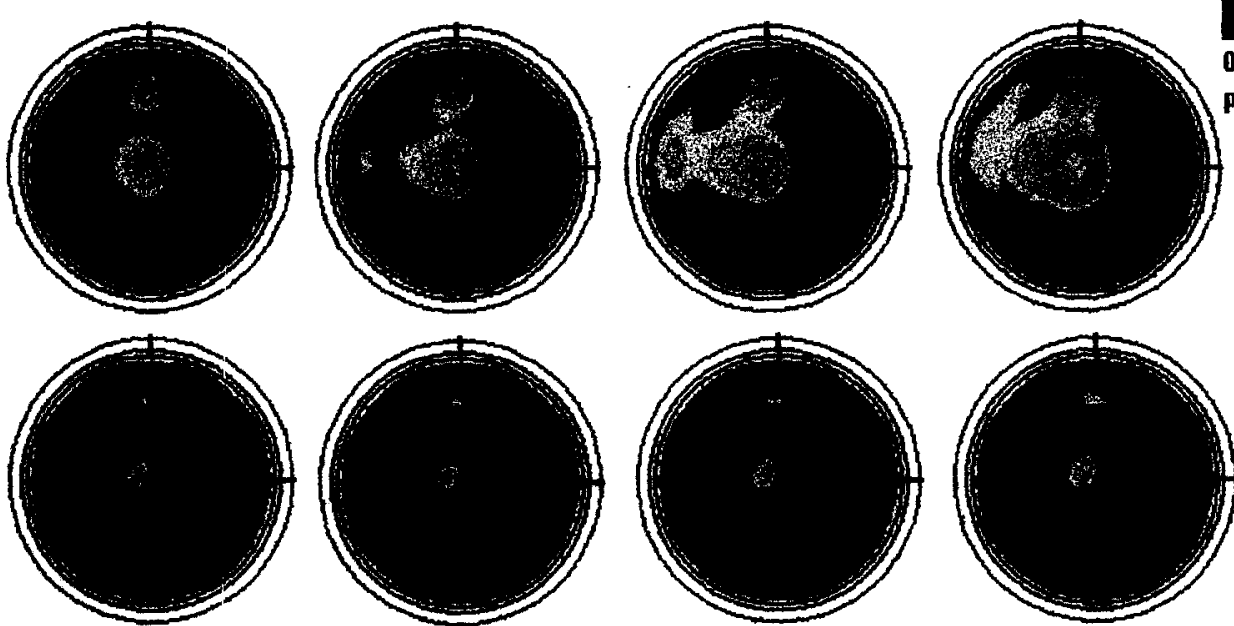

$1.8 \mathrm{nAm} / \mathrm{cm}^{2}$
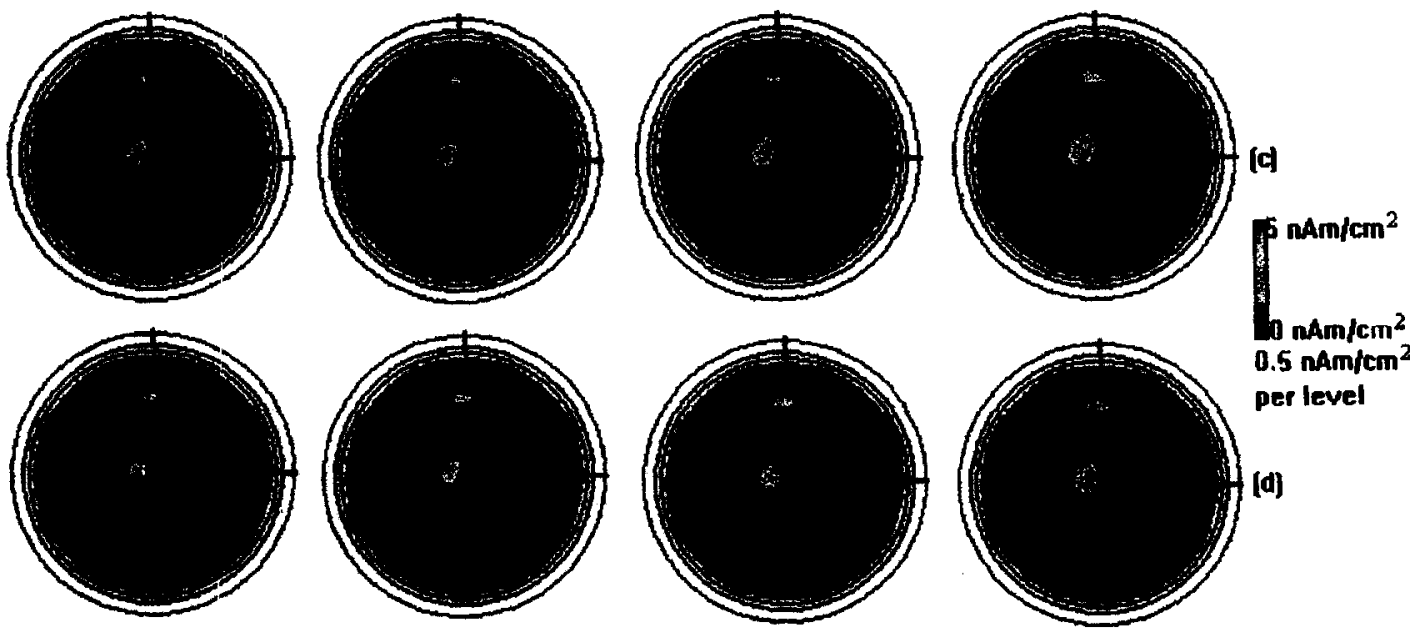

Fig. 4. Analysis of left-right averages. Current densities computed on a surface about $5 \mathrm{~mm}$ underneath the innermost shell, using linear estimation with LFN. Rows (c,d) depict results obtained with 5 sharpening iterations; $(a, c)$ show the analysis for freely selected, (b,d) for fixed movements. The $y$ axis points towards the nose and the $x$-axis towards the right ear. Note that this is a projection of the spherical reconstruction surface onto the screen. 


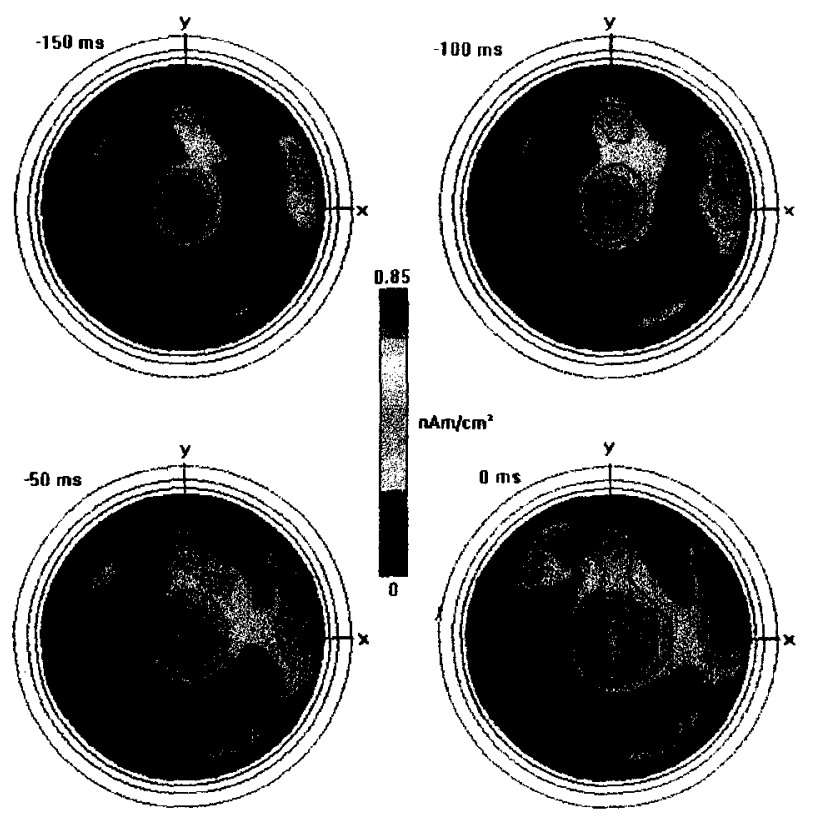

Fig. 5. Analysis of left hand RPs. Current densities computed on a surface about $5 \mathrm{~mm}$ underneath the innermost shell, using linear estimation with LFN and without iterations. The $y$-axis points towards the nose and the $x$-axis towards the right ear. Note that this is a projection of the spherical reconstruction surface onto the screen.

preceding and during voluntary movement, like the anterior cingulate cortex (cf. Deiber et al., 1991; Stephan et al., 1995). For the present, we interpret the results as in favour of an SMA contribution to the RP.

Although the linear estimation approach supports the presence of SMA activity, it differs from our earlier spatiotemporal analysis in the estimation of the relative strengths of SMA and MI contributions to the RP. In the present results, SMA activity is far dominant over the MI activity, whereas they are of more equal amplitude with spatiotemporal models (Praamstra et al., 1996). Given the evidence from intracranial recordings of the RP (Ikeda et al., 1992) and functional imaging results (e.g. Deiber et al., 1991; Shibasaki et al., 1993), linear estimation seems to overestimate SMA activity at the expense of the MI contribution. This might partially explain why the difference between the potentials preceding fixed and freely selected movements cannot, on the basis of the present results, as clearly be attributed to the SMA, as on the basis of the spatiotemporal model. The overestimation of activity located in the midline is not so remarkable in view of the distribution of the RP. Moreover, experience with spatiotemporal dipole source analysis shows that it is often necessary to impose symmetry constraints on a pair of dipoles representing the bilateral $\mathrm{MI}$, in order to counteract a tendency of sources to assume locations near the midline. Symmetry constraints were applied in most of the spatiotemporal dipole source analyses that have been proposed (Bötzel et al., 1993; Toro et al., 1993; Böcker et al., 1994; Praamstra et al., 1996). With respect to the question of how the diverging results regarding activity arising from the midline should be explained, we have argued (Praamstra et al., 1996) that several spatiotemporal source models that have been proposed do not adequately separate MI and SMA contributions to the RP. Separation may be especially difficult because of the similar time course of their activities, as suggested by intracranial recordings (Ikeda et al., 1992).

Although we suspect that SMA activity is overestimated at the expense of MI activity, in another respect there is no gross misrepresentation of MI activity. We found ipsilateral MI activity with left-hand movements, but only contralateral activity with right-hand movements. This is remarkably consistent with several recent reports noting left hemisphere involvement in the control of contra- and ipsilateral hand movements in righthanders (Kim et al., 1993; Rao et al., 1993). Somewhat speculatively, one might hypothesize that the parietal focus of activity, that was only noted with left hand movements, is also related to differences in the control of left and right hand movements.

To summarize the comparison with previously proposed generator models of the RP, we consider the present results to be in favour of an SMA contribution to the scalp-recorded potentials, thus supporting the spatiotemporal dipole source analysis of the same data (Praamstra
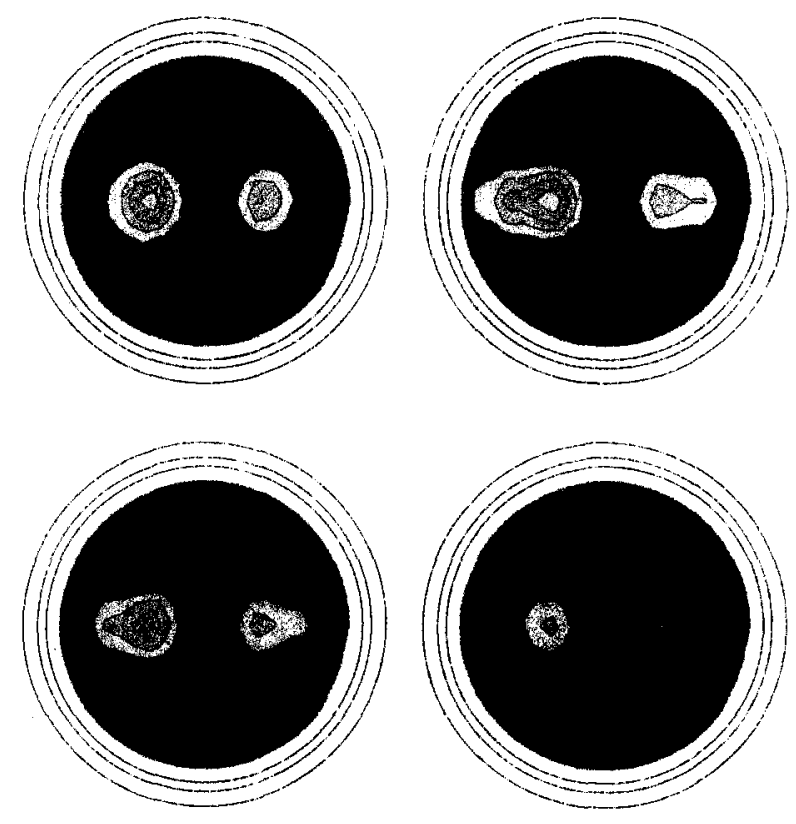

Fig. 6. Reconstructions from EEGs that were simulated from bilateral symmetrical sources at an angular position between the electrodes C3 and $\mathrm{C} 1$ for the left and $\mathrm{C} 4$ and $\mathrm{C} 2$ for the right hemisphere, and a depth of $5 \mathrm{~mm}$ underneath the innermost shell of the volume conductor model. The reconstructions are viewed from the top, the nose is pointing upwards. (a) Reconstruction of two dipoles in $z$ direction. (b) Reconstruction of two dipoles with equal components in $x$ and $z$ directions. (c) Reconstruction of two dipoles with components in $x, y$, and $z$ directions. (d) Reconstruction of two dipoles with equal components in $y$ and $z$ direction. 
et al., 1996). It should be noted, however, that the linear estimation also yielded results that are not readily interpretable. Clearly, such features should be further investigated on the basis of data with better spatial resolution (see below).

\subsection{Linear estimation verisus spatiotemporal dipole source analysis}

Reconstruction of the sources of EEG or MEG does not provide unique solutions. Different methods apply different models (also referred to as constraints) to the potential generators, which leads to different results. However, experience has shown that there may be common features between the results of different methods (e.g. approximate locations, the number of active areas or sources, source orientations). Obviously, the features shared by solutions from different analysis approaches are more likely to be part of the true generator configuration than incidental features. It is, therefore, useful to apply different source analysis approaches to the same problem, in order to identify those features. With respect to spatiotemporal dipole source analysis and linear estimation, we found with both methods activity at midline and lateral locations clearly discriminated. The convergence provides meaningful support for the spatiotemporal analysis, which involved a rather elaborate procedure to obtain a plausible reconstruction of SMA activity.

One of the main differences between linear estimation and spatio-temporal dipole fitting (STD) is that STD allows the user to define constraints that reduce the space of possible solutions and, thereby, help to guide the solution into the desired direction. This is quite often a clear advantage, but it also introduces a certain amount of subjectivity into the results. This is illustrated by the spatiotemporal dipole source models that have been proposed for the LFN (Bötzel et al., 1993; Toro et al., 1993; Böcker et al., 1994; Tarkka, 1994; Praamstra et al., 1996). We therefore consider it valuable to have a method at our disposal, i.e. linear estimation, that offers fewer possibilities to guide the solution. Linear estimation may be used in 3 ways: (1) to validate an STD solution without invoking the prior knowledge that was used to obtain the STD solution; (2) to produce an initial guess to provide the researcher with information to guide the STD procedure; or (3) to get stand-alone solutions that cannot, especially if the sources are extended, be achieved by dipole fit analysis.

The advantage that linear estimation does not impose a priori constraints on the solution (in principle any current distribution could be modelled) has to be traded for a rather low resolution of the reconstruction (analysis of the present data involved fitting of more than 1300 parameters to only 28 measurements). Because of the smoothing properties of the minimum norm condition, the reconstruction of more dipoles than electrode sites works as a sort of interpolation. To obtain sharper and more detailed images, one could: (1) use more electrodes (high resolution EEG), (2) use MEG and EEG simultaneously, (3) geometrically restrict the source space, e.g. by using functional magnetic resonance imaging or PET information, or (4) modify the algorithm in such a way that the time slices are not fitted independently any more. All these measures would improve the ratio between unknown source parameters and measurement values.

In conclusion, we believe that linear estimation can be developed into a standard tool for the reconstruction of brain activity that is in some respects superior to dipole fit methods. It is able to model any current source distribution and give us an impression of the underlying source configuration, even if we don't have a priori information, while it is very flexible in using additional information if available.

\section{References}

Böcker, K.B., Brunia, C.M. and Cluitmans, P.J. A spatiotemporal dipole model of the readiness potential in humans, I: finger movement. Electroenceph. clin. Neurophysiol., 1994, 91: 275-285.

Bötzel, K., Plendl, H., Paulus, W. and Scherg, M. Bereitschaftspotential: is there a contribution from the supplementary motor area? Electroenceph. clin. Neurophysiol., 1993, 89: 187-196.

Dale, A.M. and Sereno, M.I. Improving localization of cortical activity by combining EEG and MEG with MRI cortical surface reconstruction: a linear approach. J. Cogn. Neurosci., 1993, 5(2): 162176.

Deecke, $\mathbf{L}$. Bereitschaftspotential as an indicator of movement preparation in supplementary motor area and motor cortex. In: R. Porter (Ed.), Motor Areas of the Cerebral Cortex. Wiley, Chichester, 1987, pp. 231-245.

Deiber, M.P., Passingham, R.E., Colebatch, J.G., Friston, K.J., Nixon, P.D. and Frackowiak, R.S.J. Cortical areas and the selection of movement: a study with positron emission tomography. Exp. Brain Res., 1991, 81: 393-402.

Fuchs, M., Kullmann, W.H. and Dössel, O. Functional imaging of neuronal brain activities: overlay of distributed current density images and morphological MR images. Eur. Radiol., 1993, 3: 4143.

George, J.S., Aine, C.J., Mosher, J.C., Schmidt, D.M., Ranken, D.M., Schlitt, H.A., Wood, C.C., Lewine J.D., Sanders, J.A. and Belliveau, J.W. Mapping function in the human brain with magnetoencephalography, anatomical magnetic resonance imaging, and functional magnetic resonance imaging. J. Clin. Neurophysiol., 1995, 12(5): 406-431.

Gorodnitsky, I., George, J.S., Schlitt, H.A. and Lewis, P.S. A weighted iterative algorithm for neuromagnetic imaging. In: Satellite Symposium on Neuroscience and Technology, 14th Annual International Conference of the IEEE Engineering in Medicine and Biology Society (Abstracts), Lyon, 1992.

Ikeda, A., Lüders, H.O., Burgess, R.C. and Shibasaki, H. Movementrelated potentials recorded from supplementary motor area and primary motor area: role of supplementary motor area in voluntary movements. Brain, 1992, 115: 1017-1043.

Kim, S., Ashe, J., Hendrich, K., Ellermann, J.M., Merkle, H., Ugurbil, I. and Georgopoulos, A.P. Functional magnetic resonance imaging of the motor cortex: hemispheric asymmetry and handedness. Science, 1993, 261: 615-617.

Praamstra, P., Stegeman, D.F. Horstink, H.W., Brunia, C.H. and Cools, A.R. Movement-related potentials preceding voluntary movement 
are modulated by the mode of movement selection. Exp. Brain Res., 1995, 103: 429-439.

Praamstra, P., Stegeman, D.F. Horstink, M.W.I.M. and Cools, A.R. Dipole source analysis suggests selective modulation of the supplementary motor area contribution to the readiness potential. Electroenceph. clin. Neurophysiol., 1996, in press.

Rao, S.M., Binder, J.R., Hammeke, T.A., Yetkin, F.Z., Jesmanowicz, A., Lisk, L.M., Morris, G.L., Mueller, W.M., Estkowski, L.D., Wong, E.C., Haughton, V.M. and Hyde, J.S. Functional magnetic resonance imaging of complex human movements. Neurology, 1993, 43: 2311-2318.

Scherg, M. and Berg, P. Use of prior knowledge in brain electromagnetic source analysis. Brain Topogr., 1991, 4(2): 143-150.

Shibasaki, H., Sadato, N., Lyshkow, H., Yonekera, Y., Nagamine, M., Honda, T., Suwazono, S., Magata, Y., Ikeda, A., Miyazaki, M. and
Fukuyama, H. Both primary motor cortex and supplementary motor area play an important role in complex finger movement. Brain, 1993, 116: 1387-1398.

Stephan, K.M., Fink, G.R., Passingham, R.E., Silberzweig, D., CeballoBaumann, A.O., Flith, C.D. and Frackowiak, R.S.J. Functional anatomy of the mental representation of upper extremity movements in healthy subjects. J. Neurophysiol., 1995, 73: 373-386.

Tarkka, I.M. Electrical source localization of human movement-related cortical potentials. Int. J. Psychophysiol., 1994, 16: 81-88.

Toro, C., Matsumoto, J., Deutschl, G., Roth, B.J. and Hallet, M. Source analysis of scalp-recorded movement related electrical potentials. Electroenceph. clin. Neurophysiol., 1993, 86: 167-175.

Wang, J.-Z. MNLS inverse discriminates between neuronal activity on opposite walls of a simulated sulcus of the brain. IEEE Trans. Biomed. Eng., 1994, 41(5): 470-479. 